\title{
Eficácia do herbicida amicarbazone no controle em pós-emergência de espécies de corda-de-viola (Ipomoea grandifolia e Merremia cissoids)
}

\author{
Efficacy of amicarbazone herbicide on post-emergence control in morningglory \\ species (Ipomoea grandifloia and Merremia cissoides)
}

\section{Lucas Perim $^{1}$; Roberto Estevão Bragion Toledo ${ }^{2}$; Eduardo Negrisoli ${ }^{3}$; Marcelo Rocha Corrêa ${ }^{3}$; Caio Antônio Carbonari ${ }^{1}$; Caio Vitagliano Santi Rossi ${ }^{4}$; Edivaldo Domigues Velini ${ }^{5}$}

\begin{abstract}
Resumo: Diferentes espécies de plantas daninhas estão presentes nos canaviais brasileiros, interferindo negativamente no processo produtivo, sendo e as espécies de corda-de-viola Ipomoea sp e Merremia sp, ocupam lugar de destaque, principalmente em áreas de cana crua. Portanto, o objetivo do trabalho foi verificar a eficácia do herbicida amicarbazone no controle de Ipomoea grandifolia e Merremia cissoides, aplicado em pós-emergência e comparado ao herbicida 2,4-D. Para tanto, conduziu-se um experimento em condições de casa de vegetação, em vasos, com Latossolo Vermelho distrófico ( $\mathrm{LVd}$ ), semeados com as plantas daninhas. O delineamento experimental utilizado foi o inteiramente casualizado com cinco tratamentos e quatro repetições, e as médias submetidas ao teste de Tukey ao nível de $5 \%$ de probabilidade. Os tratamentos foram constituídos por 700; 1050; $1400 \mathrm{~g}$ i.a. ha ${ }^{-1}$ de amicarbazone, $1612 \mathrm{~g}$ i.a. ha ${ }^{-1}$ de 2,4-D e testemunha sem aplicação. A aplicação dos tratamentos foi realizada em pós-emergência, quando as plantas daninhas encontravam-se com $15 \mathrm{~cm}$, e aos 7, 14 e 21 dias após a aplicação (DAA) e avaliou-se o controle, com coleta da parte aérea das plantas para obtenção de massa seca, aos 21 DAA. Após a coleta das plantas, realizou-se nova semeadura nos vasos com as mesmas espécies de plantas daninhas e aos 7, 14 e 21 dias após a semeadura (DAS) foram realizadas avaliações visuais de controle, com o objetivo de verificar o efeito residual dos tratamentos. O delineamento experimental utilizado foi o inteiramente casualizado com cinco tratamentos e quatro repetições, e as médias comparadas pelo teste de Tukey ao nível de $5 \%$ de probabilidade. Verificou-se que, independentemente da espécie de cordas-de-viola estudada, o herbicida amicarbazone, nas doses de 700; 1050 e 1400 g i.a. ha $^{-1}$ apresentou controle total aos 21 DAA, assim como até os 21 DAS, também se constatou excelente efeito residual para este herbicida.
\end{abstract}

Palavras-chave: Corriola, plantas daninhas, controle, efeito residual.

\footnotetext{
Abstract - Different weeds species are present in Brazilian sugar cane areas interfering negatively in productive process, and morningglory species, Ipomoea sp. and Merremia sp., are

${ }^{1}$ Eng. Agr. MSc Doutorando, Dep. de Produção Vegetal, FCA/Unesp, Fazenda Experimental Lageado, Caixa Postal 237, 18603-970, Botucatu-SP, lperim@fca.unesp.br Eng.-Agr Dr. Piracicaba-SP.

${ }^{2}$ Eng.-Agr Dr. Piracicaba-SP.

${ }^{3}$ Eng.-Agr Dr. TechField-Nupam/FCA/Unesp, Fazenda Experimental Lageado, 18603-970, Botucatu-SP.

${ }^{4}$ Eng. Agr. Dr. Uberlândia-MG.

${ }^{5}$ Professor Dr. Depto. Produção Vegetal. FCA/UNESP, Botucatu-SP, Fazenda Experimental Lageado, Caixa Postal 237, 18603-970, Botucatu-SP.
} 
outstanding, particularly in raw cane areas. This way, the objective of this study was to verify the effectiveness of amicarbazone herbicide in controlling Ipomoea grandifolia and Merremia cissoides species, applied in post emergency and compared to 2,4-D herbicide. So, it was conducted an experiment, in green house conditions, in pots, with Latossoil Red distrofic (LRd), seeded with weeds. The experimental design used was entirely randomized with five treatments and four replications, and mediums submitted to Tukey test at $5 \%$ of probability. Treatments consisted of 700, 1050, $1400 \mathrm{~g}$ i.a. ha ${ }^{-1}$ of amicarbazone, $1612 \mathrm{~g}$ i.a. ha $^{-1}$ of 2,4-D and an check. Treatments application was carried out in post-emergence, when weeds containing $15 \mathrm{~cm}$ tall, and at 7, 14 and 21 days after application (DAA) and the control was assessed, with dossel plants collect for obtaining dry mass, at 21 DAA. After collecting plants, it was conducted a new sowing in pots with the same weeds species and at 7,14 and 21 days after sowing (DAS) visual control evaluations were conducted, in order to verify the residual effect of treatments. The experimental design was entirely randomized with five treatments and four replications, and mediums submitted to Tukey test at $5 \%$ of probability. It was verified that, independent of morningglory species studied, amicarbazone herbicide at 700, 1050, $1400 \mathrm{~g}$ i.a. ha ${ }^{-1}$ doses showed total control at 21 DAA, as well as up to 21 DAS, it was also observed excellent residual effect for this herbicide.

Keywords: Morningglory, weeds, control, residual effect.

\section{Introdução}

Um dos pontos mais críticos no processo produtivo da cana-de-açúcar é, sem dúvida, a interferência negativa imposta pelas plantas daninhas, as quais podem interferir diretamente sobre a cultura, competindo diretamente por fatores limitados do meio em que convivem ou liberando aleloquímicos, ou indiretamente, sendo hospedeiras de pragas, doenças e nematóides (Pitelli, 1985). Causam, ainda, reduções na quantidade e qualidade do produto colhido, diminuindo o número de cortes economicamente viáveis do canavial (Kuva, 1999).

O conhecimento dos fatores que influenciam a atividade e a estabilidade de herbicidas no solo é de grande importância, uma vez que é possível explicar o sucesso ou o fracasso desses produtos no controle das plantas daninhas, na tolerância da cultura e na persistência destes em diferentes condições de meio ambiente (Gazziero et al., 2001).
Segundo Lorenzi (1995), a presença de plantas daninhas na lavoura de cana-de-açúcar pode acarretar aumento nos custos de produção em até $30 \%$ em cana-soca e de 15 a $25 \%$ em cana-planta. Dessa forma, o controle de plantas daninhas na lavoura canavieira reveste-se de grande importância em face das grandes extensões de áreas. Por esse fator territorial e pela grande eficácia, os herbicidas são amplamente utilizados na cultura da cana-deaçúcar (Pedrinho \& Durigan, 2001).

Segundo Toledo et al. (2004), o mecanismo de ação principal do herbicida amicarbazone é a inibição da fotossíntese das plantas daninhas, atuando na reação de Hill (fotossistema II), inibindo o transporte de elétrons e paralisando a fixação de $\mathrm{CO}_{2}$ e produção de ATP e $\mathrm{NADPH}_{2}$, os quais são elementos essenciais ao crescimento das plantas. A morte das plantas, entretanto, pode ocorrer devido a outros processos, como a peroxidação de lipídeos e proteínas, promovendo a destruição das membranas e perda de clorofila. Os autores ainda mencionam que os principais benefícios do 
amicarbazone além da excelente eficácia agronômica e o prolongado período residual no controle de plantas daninhas, sendo alternativa para catação química do capim-colonião, flexibilidade na aplicação em pré e pósemergência inicial da cultura da cana-deaçúcar, que crescerá livre da interferência das plantas daninhas, favorecendo seu rendimento de colheita e qualidade do açúcar e álcool, à medida que permite reduzir do teor de impurezas no processo industrial.

Assim como o amicarbazone, a maioria dos herbicidas utilizados na cultura da cana-deaçúcar são recomendados para aplicação em condições de pré-emergência e/ou pós emergência inicial da cultura e plantas daninhas, e desta forma o principal destino final será o solo. Portanto, estes herbicidas apresentam a dinâmica afetada por fatores relacionados às suas características físicoquímicas (solubilidade, adsortividade, volatilidade e outras). Estas propriedades interagem com as condições climáticas e edáficas, que irão determinar a disponibilidade do herbicida na solução do solo (Christoffoleti \& Ovejero, 2005).

O 2,4-D é uma auxina sintética que atua provocando distúrbios diversos (crescimento anormal de tecidos, obstrução do floema, morte do sistema radicular, epinastia das folhas, etc.), os quais levam as plantas sensíveis à morte, como é o caso das diferentes espécies de cordade-viola (Rodrigues \& Almeida, 1998). É um herbicida que em algumas condições ambientais desfavoráveis à aplicação pode apresentar problemas de deriva e volatilização, causando danos às culturas sensíveis localizadas nas proximidades. Além disso, os produtos a base de 2,4-D não apresentam efeito sobre o banco de sementes de plantas daninhas (Ashton \& Crafts, 1981).

Ainda em relação ao 2,4-D, vários estudos foram realizados no sentido de verificar o seu período residual no ambiente (Altom \& Stritzke, 1973; Foster \& McKercher, 1973; Plumb et al., 1977). Quando o produto é aplicado no solo, a molécula é degradada por processos microbiológicos (Loos, 1975; Rodrigues \& Almeida, 1995). Segundo Smith (1978), o tempo necessário para esse herbicida atingir metade da sua concentração original (meia vida) é menor que sete dias.

Assim, o objetivo deste trabalho foi avaliar a eficácia e período de efeito residual do amicarbazone no controle de espécies de corda-de-viola (Ipomoea grandifolia $e$ Merremia cissoides), quando aplicado em pósemergência e comparado ao herbicida 2,4-D.

\section{Material e métodos}

A presente pesquisa foi realizada em condições controladas de casa de vegetação no Núcleo de Pesquisas Avançada em Matologia (NUPAM), pertencente ao Departamento de Produção Vegetal da Faculdade de Ciências Agronômicas/UNESP - Botucatu-SP.

O solo utilizado como substrato foi inicialmente seco à sombra por um período de 48 horas, e posteriormente peneirado em peneira com malha de 200 mesh, antes de ser submetido à análise físico-química (Tabela 1). Com base nos resultados da análise, o solo foi corrigido e adubado e, em seguida, acondicionado em vasos de 2 litros de capacidade, procedendo-se à semeadura das espécies de plantas daninhas Ipomoea grandifolia (IAQGR) e Merremia cissoides (MRRCI). O experimento foi conduzido em delineamento inteiramente casualizado, com cinco tratamentos e quatro repetições, sendo os tratamentos e suas respectivas dosagens representados na Tabela 2 . 
Tabela 1. Resultados da análise físico-química da amostra de solo utilizado no experimento. Botucatu/SP, 2007.

\begin{tabular}{|c|c|c|c|c|c|c|c|c|c|c|}
\hline \multirow{2}{*}{ Solo } & \multirow{2}{*}{$\begin{array}{c}\mathrm{pH} \\
\mathrm{CaCl}_{2}\end{array}$} & \multirow{2}{*}{$\begin{array}{l}\text { M.O. } \\
\mathrm{g} \mathrm{dm}^{-3}\end{array}$} & \multirow{2}{*}{$\begin{array}{c}\text { P res. } \\
\mathrm{Mg} \mathrm{dm}^{-3}\end{array}$} & \multicolumn{6}{|c|}{$\mathrm{mmol}_{\mathrm{c}} \mathrm{dm}^{-3}$} & \multirow{2}{*}{$\begin{array}{l}\mathrm{V} \\
(\%)\end{array}$} \\
\hline & & & & $\mathrm{K}^{+}$ & $\mathrm{Ca}^{+2}$ & $\mathrm{Mg}^{+2}$ & $\mathrm{H}^{+}+\mathrm{Al}^{+3}$ & SB & $\mathrm{T}$ & \\
\hline LVd & 4,3 & 19 & 1 & 0,6 & 10 & 4 & 58 & 14,6 & 73 & 21 \\
\hline \multicolumn{11}{|c|}{ Granulometria (\%) } \\
\hline & \multirow{2}{*}{\multicolumn{2}{|c|}{ Argila }} & \multirow{2}{*}{\multicolumn{2}{|c|}{ Limo }} & & Areia & & \multirow{2}{*}{\multicolumn{3}{|c|}{ Classe textural }} \\
\hline & & & & & Fina & Média & Grossa & & & \\
\hline & \multicolumn{2}{|c|}{20} & \multicolumn{2}{|l|}{4} & 22,9 & 35,7 & 17,4 & \multicolumn{3}{|c|}{ média } \\
\hline
\end{tabular}

Fonte: Realizada pelo Departamento de Solos - FCA/UNESP - Botucatu.

A aplicação dos herbicidas foi realizada através de um equipamento instalado no laboratório do NuPAM, o qual é constituído de uma estrutura metálica com $3 \mathrm{~m}$ de altura por 2 $\mathrm{m}$ de largura, que permite acoplamento de um "carrinho" suspenso a 2,5 m de altura. A esse carrinho encontram-se duas barras de pulverização, uma responsável pelo sistema de simulação de chuva e a outra pelo sistema de pulverização de defensivos agrícolas, as quais se deslocam por uma área útil de $6 \mathrm{~m}^{2}$ no sentido do comprimento do equipamento. $\mathrm{O}$ tracionamento de ambas as barras é realizado por meio de correntes e engrenagens, com auxílio de um motor elétrico, cujo ajuste é dado por um modulador de frequência, permitindo a obtenção de velocidade constante previamente determinada. O sistema de pulverização tem funcionamento independente do sistema de simulação de chuva, apesar de ambos compartilharem o mesmo equipamento e funções de controle.

Tabela 2. Tratamentos e respectivas dosagens utilizadas no experimento. Botucatu/SP, 2007.

\begin{tabular}{lcc}
\hline \multicolumn{1}{c}{ Tratamentos } & Dose dos herbicidas $\left(\mathrm{g}\right.$ i.a. ha $\left.{ }^{-1}\right)$ & Dose de Lanzar v/v (\%) \\
\hline 1. Amicarbazone & 1400 & 0,5 \\
2. Amicarbazone & 1050 & 0,5 \\
3. Amicarbazone & 700 & 0,5 \\
4. 2,4 - D & 1612 & 0,5 \\
5. Testemunha & - & - \\
\hline
\end{tabular}

A barra de pulverização foi constituída por quatro pontas de pulverização XR 11002 VS, espaçadas entre si em $0,5 \mathrm{~m}$ e posicionadas a $0,5 \mathrm{~m}$ de altura em relação à superfície das unidades experimentais. O sistema de pulverização foi operado sob pressão constante de 1,5 bar, pressurizado por ar comprimido, com velocidade de deslocamento de $3,6 \mathrm{~km} \mathrm{~h}^{-1}$, que correspondeu a 45,0 Hertz no modulador de frequiência, e consumo de calda correspondente a $200 \mathrm{~L} \mathrm{ha}^{-1}$.

O controle das espécies de plantas daninhas foi determinado por meio de uma escala visual e percentual de notas, variando de 0 a $100 \%$, onde zero consistiu em nenhum controle e $100 \%$ o controle total das espécies daninhas. As avaliações foram realizadas até o 
desaparecimento e/ou estabilização dos Malezas (ALAM, 1974), a qual varia de 1 sintomas de fitointoxicação (SBCPD, 1995), (nenhum a pobre) a 6 (excelente controle) sendo também correlacionadas com a escala de (Tabela 3 ).

notas da Asociación Latino Americana de

Tabela 3. Escala de notas da ALAM (1974) utilizada para avaliação da porcentagem de controle das plantas daninhas estudadas.

\begin{tabular}{cc}
\hline Porcentagem $(\%)$ & Grau de controle \\
\hline $0-40$ & Nenhum a pobre (1) \\
$41-60$ & Regular (2) \\
$61-70$ & Suficiente (3) \\
$71-80$ & Bom (4) \\
$81-90$ & Muito Bom (5) \\
$91-100$ & Excelente (6) \\
\hline
\end{tabular}

Avaliou-se a porcentagem de controle das plantas daninhas aos 7, 14, 21 dias após a aplicação (DAA), a biomassa seca da parte aérea das plantas aos 21 DAA, assim como o efeito residual de controle das mesmas espécies de plantas daninhas 7, 14 e 21 dias após a nova semeadura (DAS), ou seja, 28, 35 e 42 DAA.

Os dados originais foram submetidos à análise de variância e suas médias ao teste de Tukey ao nível de $5 \%$ de probabilidade.

\section{Resultados e discussão}

Nas Tabelas 4 e 5 estão apresentadas os níveis de controle médios e suas respectivas notas pelas ALAM, para as espécies de plantas daninhas nas diferentes épocas de avaliação. Os sintomas de danos caracterizados pelo controle foram observados a partir dos 7 DAA em todos os tratamentos avaliados. O amicarbazone, na dose de $1400 \mathrm{~g}$ i.a. ha ${ }^{-1}$ proporcionou excelente controle (nota 5) tanto para Ipomoea grandifolia como para Merremia cissoides aos 14 DAA. Ao contrário das dosagens de 1050 e 700 g i.a. ha ${ }^{-1}$, as quais apresentaram controles suficientes (nota 3) para I. grandifolia e bom (nota 4) para $M$. cissoides. A aplicação de 2,4- D (1612 g i.a. ha$\left.{ }^{1}\right)$ proporcionou controle excelente (nota 6) sobre as duas espécies testadas aos 14 DAA. O mesmo comportamento foi observado por Toledo et al. (2009), onde para aplicação de amicarbazone sobre a palha ou solo na cultura da cana-de-açúcar, apresentou excelente controle de I. grandifolia e M. cissoides a partir dos 14 DAA, concluído ser uma excelente alternativa para o manejo destas espécies de plantas daninhas em cana crua.

No entanto, todos os tratamentos com amicarbazone $\left(700 ; 1050 ; 1400\right.$ g i.a. ha $\left.{ }^{-1}\right)$ e 2,4-D (1612 g i.a ha $\left.{ }^{-1}\right)$ apresentaram excelentes níveis de controle $(100 \%)$ para I. grandifolia e M. cissoides, a partir dos 21 DAA. 
Tabela 4. Porcentagem média e notas de controle de Ipomoea grandifolia (IAOGR), segundo Escala da ALAM (1974). Botucatu/SP. 2007.

\begin{tabular}{|c|c|c|c|c|c|c|}
\hline Tratamentos & 7 DAA & ALAM & 14 DAA & ALAM & 21 DAA & ALAM \\
\hline 1. Amicarbazone (1400 g i.a. ha $\left.^{-1}\right)$ & $45,00 \mathrm{a}$ & 2 & $88,25 \mathrm{ab}$ & 5 & $100,00 \mathrm{a}$ & 6 \\
\hline 2. Amicarbazone ( $1050 \mathrm{~g}$ i.a. $\left.\mathrm{ha}^{-1}\right)$ & $37,50 \mathrm{a}$ & 1 & $71,25 \mathrm{ab}$ & 3 & $100,00 \mathrm{a}$ & 6 \\
\hline 3. Amicarbazone (700 g i.a. ha $^{-1}$ ) & $25,00 \mathrm{a}$ & 1 & $68,75 b$ & 3 & $100,00 \mathrm{a}$ & 6 \\
\hline 4. 2,4-D (1612 g i.a. $\left.\mathrm{ha}^{-1}\right)$ & $50,00 \mathrm{a}$ & 2 & $94,50 \mathrm{a}$ & 6 & $100,00 \mathrm{a}$ & 6 \\
\hline
\end{tabular}

\section{Testemunha}

Médias seguidas das mesmas letras não diferem estatisticamente entre si pelo teste de Tukey a 5\% de probabilidade

Tabela 5. Porcentagem média e notas de controle de Merremia cissoides (MRRCI), segundo Escala da ALAM (1974). Botucatu/SP. 2007.

\begin{tabular}{|c|c|c|c|c|c|c|}
\hline Tratamentos & 7 DAA & ALAM & 14 DAA & ALAM & 21 DAA & ALAM \\
\hline 1. Amicarbazone (1400 g i.a. ha $\left.^{-1}\right)$ & $23,75 \mathrm{ab}$ & 1 & $90,50 \mathrm{a}$ & 5 & $100,00 \mathrm{a}$ & 6 \\
\hline 2. Amicarbazone (1050 g i.a. ha $\left.^{-1}\right)$ & $26,25 \mathrm{ab}$ & 1 & $73,25 \mathrm{a}$ & 4 & $100,00 \mathrm{a}$ & 6 \\
\hline 3. Amicarbazone (700g i.a. ha $^{-1}$ ) & $11,25 b$ & 1 & $73,00 \mathrm{a}$ & 4 & $100,00 \mathrm{a}$ & 6 \\
\hline 4. 2,4-D (1612 $\left.\mathrm{g}_{\text {i.a. }} \mathrm{ha}^{-1}\right)$ & $37,50 \mathrm{a}$ & 1 & $91,50 \mathrm{a}$ & 6 & $100,00 \mathrm{a}$ & 6 \\
\hline 5. Testemunha & - & - & - & - & - & - \\
\hline
\end{tabular}

Médias seguidas das mesmas letras não diferem estatisticamente entre si pelo teste de Tukey a 5\% de probabilidade

Todos os tratamentos resultaram na redução da biomassa seca das plantas daninhas, como se pode observar na Tabela 6. Para $I$. grandifolia, o amicarbazone, nas doses de 1400 e $1050 \mathrm{~g}$ i.a. ha ${ }^{-1}$ (tratamentos 1 e 2), apresentaram uma resposta maior na redução da biomassa seca em 21 DAA, quando comparado estatisticamente a testemunha. Já para $M$. cissoides todos os tratamentos responderam na mesma uniformidade de redução de biomassa seca aos 21 DAA.

Nas avaliações de residual dos herbicidas nas unidades experimentais (tabela 7), observou-se que aos 21 dias após a nova semeadura (DAS), ou seja, 42 DAA, todos os tratamentos com amicarbazone apresentaram excelente efeito residual, ou seja, 97,7 a 100\% de controle das espécies $I$. grandifolia e $M$. cissoides (nota 6), enquanto que o tratamento com 2,4-D não apresentou efeito residual significativo $(30 \%)$, fato esse que corrobora com Toledo et al. (2004), os quais relataram que o amicarbazone é absorvido pelo sistema radicular e translocado via xilema. No entanto, o predomínio da absorção foliar ocorre quando aplicado em pós-emergência, promovendo sua ação de contato. Ainda segundo Costa (2001), qual relata que, para exercerem sua atividade sobre as plantas infetantes, os herbicidas residuais precisam ser introduzidos diretamente no solo, proporcionado pelo carreamento através da água de chuva, em função da solubilidade do produto. 
Tabela 6. Biomassa seca da parte aérea (g) das plantas daninhas I. grandifolia (IAOGR) e Merremia cissoides (MRRCI) aos 21 DAS. Botucatu/SP. 2007.

\begin{tabular}{|c|c|c|}
\hline \multirow{2}{*}{ Biomassa Seca $(g)$} & \multicolumn{2}{|c|}{21 DAS } \\
\hline & IAQGR & MRRCI \\
\hline 1. Amicarbazone(1400 g i.a. ha $\left.^{-1}\right)$ & $1,75 \mathrm{a}$ & $3,00 \mathrm{a}$ \\
\hline 2. Amicarbazone (1050 g i.a. ha $\left.^{-1}\right)$ & $2,00 \mathrm{ab}$ & $4,00 \mathrm{a}$ \\
\hline 3. Amicarbazone (700g i.a. ha $\left.{ }^{-1}\right)$ & $2,75 \mathrm{ab}$ & $3,75 \mathrm{a}$ \\
\hline 4. 2,4-D (1612 g i.a. $\left.\mathrm{ha}^{-1}\right)$ & $1,75 \mathrm{a}$ & $2,50 \mathrm{a}$ \\
\hline 5. Testemunha & $4,00 \mathrm{~b}$ & $7,50 \mathrm{~b}$ \\
\hline $\mathrm{CV} \%$ & 36,70 & 22,22 \\
\hline DMS & 2,02 & 2,07 \\
\hline
\end{tabular}

Médias seguidas das mesmas letras não diferem estatisticamente entre si pelo teste de Tukey a 5\% de probabilidade

Tabela 7. Porcentagem média e notas segundo a Escala da ALAM (1974) do controle do período residual sobre Ipomoea grandifolia e Merremia Cissoides aos 7,14 e 21 DAS.

\begin{tabular}{|c|c|c|c|c|c|c|c|c|c|c|c|c|}
\hline \multirow{2}{*}{ TRATAMENTOS } & \multicolumn{4}{|c|}{7 DAS } & \multicolumn{5}{|c|}{14 DAS } & \multicolumn{3}{|c|}{$21 \mathrm{DAS}$} \\
\hline & IAQGR & & MRRC & & IAQGI & & MRRCI & & IAQGR & & MRRCI & \\
\hline 1. Amicarbazone(1400 g i.a. ha $\left.{ }^{-1}\right)$ & $83,5 \mathrm{a}$ & 5 & $60,0 \mathrm{a}$ & 2 & $88,7 \mathrm{~b}$ & 5 & $72,5 b$ & 4 & $100 \mathrm{a}$ & 6 & $97,7 \mathrm{a}$ & 6 \\
\hline 2. Amicarbazone ( $1050 \mathrm{~g}$ i.a. $\left.\mathrm{ha}^{-1}\right)$ & $92,5 \mathrm{a}$ & 6 & $85,0 \mathrm{a}$ & 5 & $97,0 \mathrm{a}$ & 6 & $97,5 \mathrm{a}$ & 6 & $100 \mathrm{a}$ & 6 & $100 \mathrm{a}$ & 6 \\
\hline 3. Amicarbazone (700g i.a. ha $\left.{ }^{-1}\right)$ & $91,2 \mathrm{a}$ & 6 & $83,7 \mathrm{a}$ & 5 & $94,0 \mathrm{a}$ & 6 & $99,5 \mathrm{a}$ & 6 & $100 \mathrm{a}$ & 6 & $100 \mathrm{a}$ & 6 \\
\hline 4. 2,4-D (1612 g i.a. $\left.\mathrm{ha}^{-1}\right)$ & $37,5 b$ & 1 & $13,7 \mathrm{~b}$ & 1 & $48,0 \mathrm{~b}$ & 2 & $18,7 \mathrm{c}$ & 1 & $51,3 b$ & 2 & $30,0 b$ & 1 \\
\hline 5. Testemunha & & & & & & & & & & & & \\
\hline
\end{tabular}

Médias seguidas das mesmas letras não diferem estatisticamente entre si pelo teste de Tukey a 5\% de probabilidade

\section{Conclusões}

Pelos resultados obtidos, pode-se concluir que o herbicida amicarbazone é uma excelente ferramenta para o controle em pósemergência de Ipomoea grandifolia e Merremia cissoides, principalmente como alternativa para o 2,4-D em áreas próximas a culturas sensíveis a deriva e por apresentar residual superior no controle dessas espécies de plantas daninhas chave da cultura da cana-deaçúcar.

\section{Referências}

ALTOM, J.D.; STRITZKE, J.F. Degradation of dicamba, picloram and four phenoxy herbicides in soils. Weed Science, v.21, n.1, p.556-560, 1973. 
ASHTON, F.M.; CRAFTS, A.S. Mode of action of herbicides. New York: John Wiley, $1981.525 \mathrm{p}$.

ASOCIACIÓN LATINOAMERICANA DE MALEZAS - ALAM. Recomendaciones sobre unificación de los sistemas de evaluación en ensayos de control de malezas. ALAM, v.1, n.1, p.35-38, 1974.

CHRISTOFFOLETI, P.J.; OVEJERO, R.F.L. Dinâmica dos herbicidas aplicados ao solo na cultura da cana-de-açúcar. Piracicaba, SP. 49 p. 2005.

COSTA, E.A.D. Efeito de adjuvantes na dinâmica de ametryn em palha de cana-deaçúcar. 2001, 81p. Tese (Doutorado em Agronomia/ Proteção de Plantas) - Faculdade de Ciências Agronômicas. Universidade Estadual Paulista, Botucatu, 2001.

FOSTER, R.K.; McKERCHER, Laboratory incubation studies of chlorophenoxyacetic acids in chernozemic soils. Soil Biology and Soil Biochemistry, v.5, n.3, p.333-337, 1973.

GAZZIERO, D.L.P. et al. Comportamento do girassol quando cultivado em área tratada com o herbicida 2,4-D. Planta Daninha, v.19, n.1, p.127-133, 2001.

KUVA, M.A. Efeito de períodos de convivência e de controle das plantas daninhas na cultura da cana-de-açúcar (Saccharum spp.) no estado de São Paulo. 1999. 74 f. Dissertação (Mestrado em Fitotecnia) Escola Superior de Agricultura "Luiz de Queiroz", Piracicaba, 1999.

LOOS, M.A. Phenoxyalkanoic acids. In: KEARNEY, P.C., KAUFMAN, D.D. Herbicides. New York: Marcel Dekker, 1975. p.1-28.

LORENZI, H. Plantas daninhas na cultura da cana-de-açúcar: Plantas daninhas na lavoura do nordeste brasileiro. In: ENCONTRO
TÉCNICO GOAL, CANA-DE-AÇÚCAR, 4., 1995, Recife. Anais... Recife: 1995.

PEDRINHO, A.F.F.; DURIGAN, J.C. Controle de capim-colonião na cultura da cana-deaçúcar com herbicidas aplicados em préemergência. Revista Brasileira de Herbicidas, v.2, n.3, p.125-131, 2001.

PITELLI, R.A. Interferência de plantas daninhas em culturas agrícolas. Informe Agropecuário, v.11, n.129, p.16-27,1985.

PLUMB, T.R. et al. Persistence of 2,4-D and 2,4,5 T in chaparral soil vegetation. Bulletin of Environmental Contamination and Toxicology, v.17, n.4, p.1-8, 1977.

RODRIGUES, B.N.; ALMEIDA, F. S. Guia de herbicidas. Londrina: 1998. 648 p.

SMITH, A.E. Relative persistence of di-and trichlorophenoxyalkanoic acid herbicides in Saskatchewan soils. Weed Research, v.18, n.1, p. 275- 279, 1978.

SOCIEDADE BRASILEIRA DA CIÊNCIA DAS PLANTAS DANINHAS - SBCPD. Procedimentos para instalação, avaliação e análise de experimentos com herbicidas. Londrina: SBCPD, 1995. 42p.

TOLEDO, R.E.B. et al. Dinamic (Amicarbazone), novo herbicida seletivo para o controle de plantas daninhas em pré e pósemergência na cultura da cana-de-açúcar. In: CONGRESSO BRASILEIRO DA CIÊNCIA DAS PLANTAS DANINHAS, 24. 2004. Resumos. São Pedro: 2004. 245 p.

TOLEDO, R.E.B. et al. Eficácia do herbicida amicarbazone aplicado sobre a palha ou no solo no controle de plantas daninhas na cultura da cana-de-açúcar. Planta Daninha, v.27, n.2, p. 319-326, 2009. 